\title{
Prostate cancer-specific survival among warfarin users in the Finnish Randomized Study of Screening for Prostate Cancer
}

\author{
Pete T. T. Kinnunen ${ }^{1 *}$ (D), Teemu J. Murtola ${ }^{1,2}$, Kirsi Talala ${ }^{3}$, Kimmo Taari ${ }^{4}$, Teuvo L. J. Tammela ${ }^{1,2}$ and Anssi Auvinen ${ }^{5}$
}

\begin{abstract}
Background: Venous thromboembolic events (VTE) are common in cancer patients and associated with higher mortality. In vivo thrombosis and anticoagulation might be involved in tumor growth and progression. We studied the association of warfarin and other anticoagulant use as antithrombotic medication and prostate cancer (PCa) death in men with the disease.

Methods: The study included 6,537 men diagnosed with PCa during 1995-2009. Information on anticoagulant use was obtained from a national reimbursement registry. Cox regression with adjustment for age, PCa risk group, primary therapy and use of other medication was performed to compare risk of PCa death between warfarin users with 1) men using other types of anticoagulants and 2) non-users of anticoagulants. Medication use was analyzed as a time-dependent variable to minimize immortal time bias.

Results: In total, 728 men died from PCa during a median follow-up of 9 years. Compared to anticoagulant nonusers, post-diagnostic use of warfarin was associated with an increased risk of PCa death (overall HR 1.47, 95\% CI 1. 13-1.93). However, this was limited to low-dose, low-intensity use. Otherwise, the risk was similar to anticoagulant non-users. Additionally, we found no risk difference between warfarin and other types of anticoagulants.

Pre-diagnostic use of warfarin was not associated with the risk of PCa death.

Conclusions: We found no reduction in risk of PCa death associated with warfarin use. Conversely, the risk was increased in short-term use, which is probably explained by a higher risk of thrombotic events prompting warfarin use in patients with terminal PCa.
\end{abstract}

Keywords: Prostate cancer, Warfarin, Anticoagulant, Survival, Cohort

\section{Background}

Venous thromboembolism (VTE) has been proposed as prognostic factor in prostate cancer (PCa). VTE is common in cancer patients and associated with poor prognosis, risk of death is 8 -fold higher in cancer patient with VTE [1-3]. This applies to PCa as well [4]. VTE in PCa patients has been associated with more than 6-fold mortality in both symptomatic and incidental venous thromboembolic diseases [5]. Thus, anticoagulant drugs could have an impact on $\mathrm{PCa}$ prognosis by reducing deaths from VTE. Furthermore, anticoagulants, including

\footnotetext{
* Correspondence: Kinnunen.Pete.T@student.uta.fi

${ }^{1}$ University of Tampere, Faculty of Medicine and Life Sciences, Tampere,

Finland

Full list of author information is available at the end of the article
}

warfarin, have shown promising antitumor properties in vivo [6-10] mainly in lung and breast cancer.

Previous fairly small studies on anticoagulant use and PCa survival have reported differing results [11-13]. A potential explanation could be confounding by indication; patients with an advanced cancer are at increased risk for VTE [14], thus more often prescribed anticoagulants compared to people without cancer. We conducted a retrospective, population-based cohort study to assess the association of pre- and post-diagnostic use of warfarin and other anticoagulants with PCa survival in the Finnish Randomized Study of Screening for Prostate Cancer (FinRSPC) [15]. 


\section{Methods}

\section{Study cohort}

FinRSPC includes 80,458 men aged $55-67$ years at baseline (i.e. at FinRSPC randomization). After exclusion of prevalent $\mathrm{PCa}$ cases, the men were randomized during 1996-1999 either to PSA screening at four-year intervals (the screening arm) or to no intervention (the control arm). All men were followed via Finnish Cancer Registry, which covers 99\% of cancers diagnosed in Finland [16]. This study included 6,537 incident PCa cases diagnosed during 1996-2013. Clinical information included Gleason grade and TNM stage (available for $97.3 \%$ and $97.7 \%$ of the cases, respectively).

PCa cases were stratified into low/intermediate-risk and high-risk groups according to the definition of the European Association of Urology (EAU) [17]. All M1 cases were included in the high-risk group.

Information on deaths was obtained from the Statistics Finland, which assigns official causes of death based on mandatory death certificates, covering all deaths in Finland [18]. The accuracy of information on PCa deaths was ascertained by the FinRSPC cause of death committee with an excellent concordance between official causes of death and the cause of death committee assignments for PCa (kappa 0.95) [15]. Only deaths with PCa (ICD-10 code C61) as the primary cause of death were regarded as $\mathrm{PCa}$ deaths.

A sensitivity analysis with $\mathrm{PCa}$ as an intermediate cause of death was also performed.

\section{Information on anticoagulant usage}

In order to obtain information on anticoagulant drug purchases during 1995-2009, the study cohort was linked to a national medication reimbursement database maintained by the Finnish Social Insurance Institution (SII) using the unique personal identification code as the key. As a part of the national health insurance that covers all Finnish citizens, SII provides reimbursements for purchases of physician-prescribed drugs [19].The reimbursement is 50-100\% depending on the indication and severity of the condition. In Finland, anticoagulant drugs are available only through physicians' prescription, thus all anticoagulant purchases in outpatient setting are registered by the database. Drugs used during hospital inpatient periods are not registered.

All 13 anticoagulant drugs used in outpatient setting during the study period were identified based on their ATC codes (Additional file 1: Table S1). Additionally, we obtained information on cholesterol-lowering drugs, antidiabetic and antihypertensive drugs, aspirin and other NSAIDs and alpha-blockers. We also collected information on primary therapy of $\mathrm{PCa}$ (radical prostatectomy, external beam radiation therapy (EBRT), hormonal therapy or active surveillance/watchful waiting).
Other medication served as a proxy for co-morbidities, as they may influence survival [20-25].

The information from the National Care Register for Health Care maintained by the National Institute for Health and Welfare included provided diagnoses of conditions serving as indications for anticoagulant use: atrial fibrillation (ICD-10: I48), venous thromboembolism (all recorded I82 diagnoses in the study population), pulmonary embolism (ICD-10: I26.0, I26.9) and thrombocytosis. The register covers all of Finland and registers all diagnoses made during in- and out-patient hospital visits during 1996-2014, but does not cover diagnoses from primary care [26]. Additionally, we stratified the analysis by Charlson Comorbidity Score [27] calculated on basis of the registered diagnoses for subgroup analysis.

\section{Statistical analysis}

Cox proportional hazards regression was used to calculate hazard ratios $(\mathrm{HR})$ and $95 \%$ confidence intervals (CIs) for PCa death. Follow-up started at PCa diagnosis and continued until death, emigration from Finland or January $1^{\text {st }}, 2015$, whichever was first. Time metric was years and months since the PCa diagnosis.

We used two different model adjustments. The first Cox regression model was adjusted for age only and the second for age, EAU tumor risk group and other medications. The analysis was performed separately for pre-diagnostic and post-diagnostic use of warfarin and additionally for other types of anticoagulants. Separate comparisons were performed between all anticoagulant users and non-users to estimate the overall effect of anticoagulant usage, and between warfarin users and men using non-warfarin anticoagulants to estimate specific effects of warfarin with simultaneous control of confounding by indication.

For each man in the study cohort, the total annual amount of medication purchases was calculated for each calendar year, and then separately for pre- and postdiagnostic use. Standardization of doses between different anticoagulants was performed by dividing the annual total milligram amount with the standard Defined Daily Dose (DDD) as listed by the WHO [28]. Each year with registered anticoagulant purchases, regardless of the amount, was considered a year of usage. The intensity of the use was calculated by dividing the cumulative annual doses with the number of years of usage.

Pre-diagnostic medication use was analyzed as a timeindependent variable, and cumulative pre-diagnostic anticoagulant usage beginning from 1995 was stratified by tertiles. Post-diagnostic use was analyzed as a timedependent variable; the usage status and cumulative use were updated separately for each year of follow-up, beginning from the year of diagnosis. 
All men were categorized as non-users until the potential first anticoagulant purchase. After the first purchase, the status was changed into a user, which was maintained for each year with recorded purchases. Men who discontinued the purchases during the follow-up were kept as ever-users. In analysis of warfarin users compared to non-warfarin users, men were categorized as warfarin users each year with recorded warfarin purchases, even if they had used other types of anticoagulants. Only for years with recorded anticoagulant purchases without warfarin use, were they recorded as non-warfarin users.

Long-term impact of timing of post-diagnostic anticoagulant use was evaluated in lag-time analysis, where anticoagulant exposure was lagged 1 to 3 years forward from the actual year of usage i.e. its effect was ignored for that duration from the first exposure.

Competing risks analysis using Fine and Gray regression method with non- $\mathrm{PCa}$ death and major thromboembolic diseases as the competing cause of death was performed.

All statistical analyses were carried out using IBM SPSS Statistics 22.0. All statistical tests are two-sided.

\section{Results}

\section{Population characteristics}

During the median follow-up of 9 years after PCa diagnosis 2,296 men died, of whom 728 from PCa (Table 1). The median follow-up from the diagnosis to PCa death was 3.9 years among men with no post-diagnostic anticoagulant use, 4.8 years among warfarin users and 5.8 years in users of other anticoagulants. High-grade cancers were almost equally distributed in these subcategories. Distribution of background characteristics are presented in Table 1.

\section{Risk of PCa death by pre-diagnostic use of warfarin and other anticoagulants}

In general, when compared to anticoagulant non-users, there was no clear association between pre-diagnostic use of warfarin and PCa death in either age-adjusted or multivariable-adjusted analysis (multivariable-adjusted HR 1.15, 95\% CI 0.88-1.49). No statistically significant risk trends by cumulative amount or duration of use were observed (Table 2). However, pre-diagnostic use of warfarin for 5 years or more was associated with a borderline significant risk increase in the multivariableadjusted model (HR 1.49, 95\% CI 0.97-2.28).

When users of other anticoagulants were used as the reference group, warfarin use was not associated with PCa death. No risk trends by cumulative use were observer either (Table 2).
Risk of PCa death by post-diagnostic anticoagulant use In the analysis of post-diagnostic use of warfarin, the overall risk of $\mathrm{PCa}$ death was significantly higher among warfarin users in comparison to anticoagulant non-users (multivariable-adjusted HR 1.47, 95\% CI 1.13-1.93) (Table 3). The risk association was strongest in low-dose usage ( $<200$ DDD) (multivariable-adjusted HR 2.50, 95\% CI 1.86-3.36). Short-term (1 year or less)/low-intensity use $(<128$ DDD/year $)$ of warfarin was similarly associated with an increased risk of PCa death. As the cumulative amounts increased and the duration of usage extended to 2 years or more, the risk increase was attenuated and was no longer statistically significant.

The risk PCa death was similar for warfarin and other anticoagulants (multivariable-adjusted HR 1.01, 95\% CI $0.71-1.44)$. In the stratified analysis, short-term use of warfarin was associated with an increased risk of $\mathrm{PCa}$ death, but again the risk increase was smaller in highintensity use (Table 3).

\section{Lag-time analysis}

In order to evaluate long-term effects of warfarin use and minimize bias due to thrombotic events triggering the prescription, we performed an analysis lagging warfarin use by one, two and three years, i.e. relating it to events at least 1-3 years later than the exposure to allow for latency. The risk increase compared to anticoagulant non-users was decreased already in the analysis lagged by one year, but remained non-significantly elevated (Table 3). The risk increase noted in low-dose usage was found in the 1-year lagged analysis, but after 2-year lag-time it was only borderline significant (twoyear lag-time for $\leq 200$ DDD HR 1.34, 95\% CI 0.99-1.83). In general, extending the lag-time to two or three years did not substantially alter the results compared to oneyear lag-time analysis.

Lag-time analysis of warfarin use relative to usage of other anticoagulant drugs was not substantially altered compared with the non-lagged results. No risk increase among short-term users or decreased risk among highintensity users was found in the lagged analysis (Table 3).

\section{Subgroup analyses}

No clear risk modification by any background variable was present between pre-diagnostic warfarin use and risk PCa death (Fig. 1). The same applied also to post-diagnostic use and risk of PCa death (Fig. 2). Effect modification was not found when comparing warfarin usage to other types of anticoagulants in pre- and post-diagnostic setting (Additional file 2: Figure S1 and Additional file 3: Figure S2) 


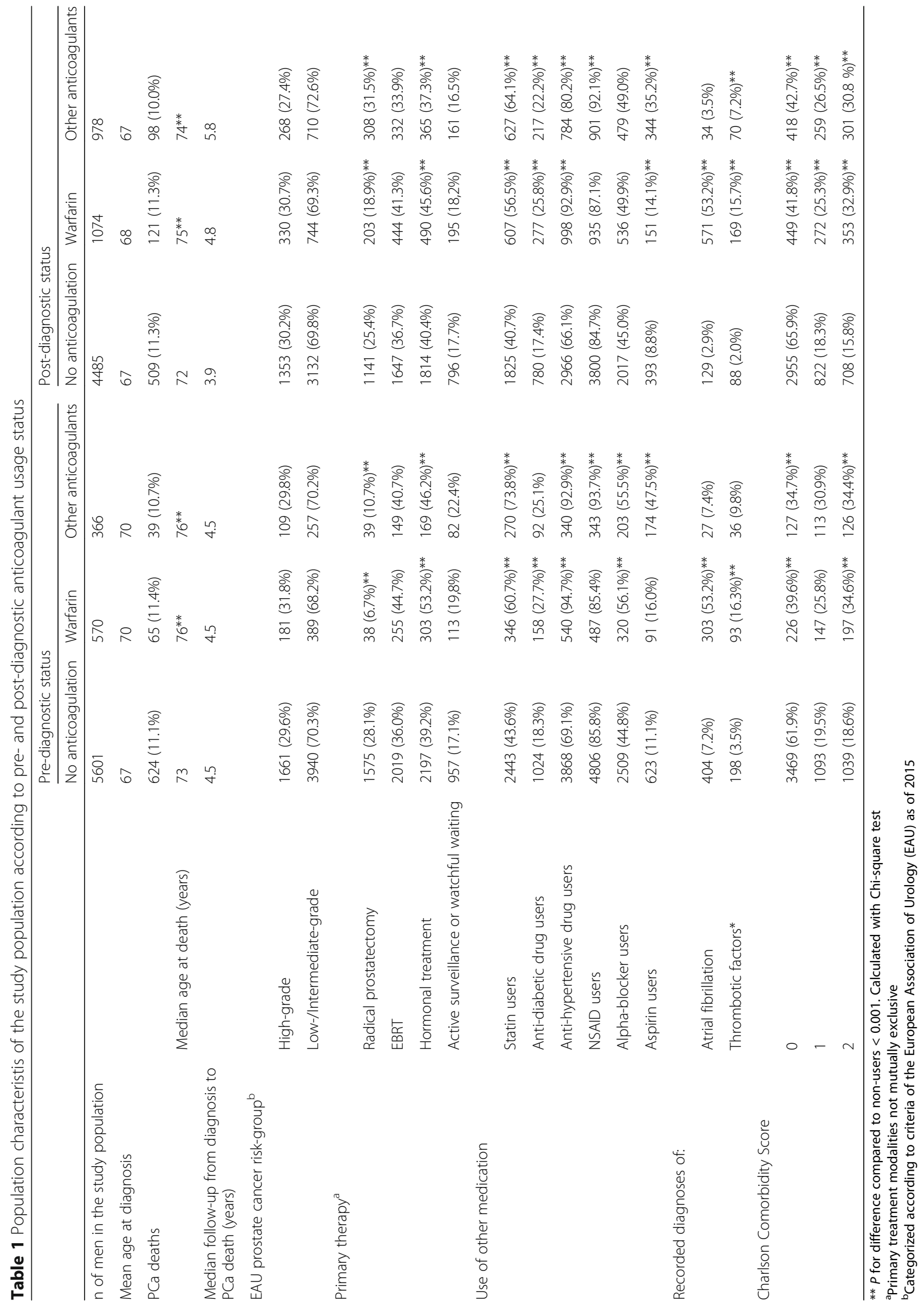


Table 2 Pre-diagnostic use of warfarin compared to anticoagulant non-users and warfarin usage in comparison with other anticoagulant drugs stratified by Defined Daily Doses (DDD), duration and intensity of usage

\begin{tabular}{|c|c|c|c|}
\hline & $\mathrm{n}$ of deaths & Age-adjusted & Multivariable-adjusted \\
\hline \multicolumn{4}{|c|}{ Warfarin compared to non-users } \\
\hline None & 624 & Ref & Ref \\
\hline Any & 65 & $1.11(0.85-1.44)$ & $1.15(0.88-1.49)$ \\
\hline \multicolumn{4}{|l|}{ Amount of warfarin use } \\
\hline$\leq 200$ DDD & 24 & $0.98(0.65-1.48)$ & $0.97(0.64-1.47)$ \\
\hline 201-796 DDD & 18 & $1.09(0.68-1.74)$ & $1.19(0.74-1.91)$ \\
\hline$>796$ DDD & 23 & $1.31(0.86-1.99)$ & $1.37(0.90-2.09)$ \\
\hline \multicolumn{4}{|c|}{ Duration of warfarin use } \\
\hline$\leq 1$ year & 20 & $0.91(0.53-1.56)$ & $0.72(0.42-1.24)$ \\
\hline $2-4$ years & 22 & $0.96(0.57-1.61)$ & $0.87(0.52-1.47)$ \\
\hline 5 or more years & 23 & $1.33(0.80-2.23)$ & $1.16(0.69-1.94)$ \\
\hline \multicolumn{4}{|l|}{ Intensity of warfarin use } \\
\hline$\leq 114$ DDD/year & 21 & $0.95(0.56-1.62)$ & $0.79(0.47-1.35)$ \\
\hline 115-200 DDD/year & 24 & $1.09(0.65-1.81)$ & $0.94(0.57-1.57)$ \\
\hline >200 DDD/year & 20 & $1.10(0.64-1.89)$ & $0.96(0.56-1.65)$ \\
\hline
\end{tabular}

Age-adjusted and multivariable-adjusted hazard ratios $(95 \% \mathrm{Cl})$ related to all PCa deaths

\section{Sensitivity analysis}

As part of sensitivity analyses, users of any anticoagulants including warfarin were compared to non-users. There was a borderline significant risk increase among pre-diagnostic users of anticoagulants (multivariable-adjusted HR 1.19, 95\% CI 0.96-1.48) (Additional file 4: Table S2).

In analysis of post-diagnostic use, the risk was increased (HR 1.58, 95\% CI 1.27-1.97), but no apparent risk trends by cumulative amount or duration were observed. In the lag-time analyses, no statistically significant risk increase was present (Additional file 4: Table S3).

No clear effect modification was observed for pre- nor post-diagnostic use of all anticoagulants (Additional file 5: Figure S3 and Additional file 6: Figure S4).

\section{Competing risk analysis}

In a Fine-Gray regression analysis with non-PCa deaths as competing cause of death, no risk difference was observed between users of warfarin and other anticoagulants (HR 0.95, 95\% CI 0.74-1.24). The findings were similar when deaths caused by pulmonary embolism or stroke were analyzed as the competing causes of death (HR 0.95, 95\% CI 0.73-1.23).

\section{Discussion}

Short-term and low-dose warfarin use after the diagnosis was associated with increased risk of PCa death compared to anticoagulant non-users. However, in analyses allowing for lag-time, the risk was attenuated after one year and disappeared in 2-year or 3-year lag-time analysis. This suggests that the risk increase occurs only for a short time period after starting drug use. We found no statistically significant risk difference between users of warfarin and other types of anticoagulants. Additionally, we found no association between pre-diagnostic use of warfarin and PCa survival. Although the indication for anticoagulant treatment did not modify the risk association, it is likely that the short-term increased risk is explained by thrombotic events as indications for anticoagulation. In epidemiological literature this phenomenon where pharmaceutical drug is prescribed for early manifestation of yet un-diagnosed disease is referred to as 'protopathic bias' [29].

Previous studies on warfarin and PCa mortality are sparse. To date, three published studies have assessed the association between PCa survival and use of vitamin $\mathrm{K}$ antagonists [11-13]. Tagalakis et al. found an increased risk of $\mathrm{PCa}$ death associated with one-year or ever-use of warfarin in after PCa diagnosis [11]. Our study is consistent with an increased risk associated with short-term use. In contrast to Tagalakis et al., we were able to evaluate cumulative amounts and intensity of use and found that high-dose or long-term use are not associated with risk increase.

In a cohort study including 12,186 men with $\mathrm{PCa}$, but no information on stage no evidence was found for an association between PCa death and pre-diagnostic use of warfarin during a mean follow-up of 3.7 years [12]. In a sensitivity analysis post-diagnostic use was associated with an increased risk of PCa death. Evaluation of cumulative amounts, duration nor intensity of use for post- 
Table 3 Post-diagnostic use of warfarin compared to anticoagulant non-users and seperately to users of other types of anticoagulants stratified by Defined Daily Doses (DDD), duration and intensity of usage

\begin{tabular}{|c|c|c|c|c|c|c|}
\hline & $\mathrm{n}$ of deaths & Age-adjusted & Multivariable-adjusted & 1-year lag-time & 2-year lag-time & 3-year lag-time \\
\hline \multicolumn{7}{|l|}{ Warfarin compared to non-users } \\
\hline None & 509 & Ref & Ref & Ref & Ref & Ref \\
\hline Any & 121 & $1.46(1.12-1.90)$ & $1.47(1.13-1.93)$ & $1.12(0.85-1.48)$ & $1.12(0.85-1.48)$ & $1.08(0.83-1.41)$ \\
\hline \multicolumn{7}{|l|}{ Amount of warfarin use } \\
\hline$\leq 200$ DDD & 63 & $2.47(1.84-3.31)$ & $2.50(1.86-3.36)$ & $1.45(1.04-2.02)$ & $1.34(0.99-1.83)$ & $1.26(0.94-1.70)$ \\
\hline 200-667 DDD & 32 & $0.87(0.53-1.42)$ & $0.88(0.54-1.46)$ & $0.85(0.52-1.41)$ & $0.76(0.44-1.32)$ & $0.76(0.43-1.36)$ \\
\hline$>667$ DDD & 26 & $0.97(0.56-1.68)$ & $1.02(0.59-1.78)$ & $1.05(0.60-1.83)$ & $1.15(0.67-1.97)$ & $1.04(0.58-1.85)$ \\
\hline \multicolumn{7}{|l|}{ Duration of warfarin use } \\
\hline$\leq 1$ year & 56 & $2.03(1.47-2.81)$ & $2.04(1.47-2.83)$ & $1.26(0.89-1.78)$ & $1.25(0.91-1.71)$ & $1.15(0.85-1.57)$ \\
\hline 2-4 years & 44 & $1.28(0.88-1.87)$ & $1.30(0.89-1.90)$ & $1.11(0.73-1.69)$ & $0.87(0.53-1.42)$ & $0.93(0.56-1.54)$ \\
\hline 5 or more years & 21 & $0.95(0.49-1.85)$ & $1.05(0.54-2.04)$ & $1.06(0.56-1.99)$ & $1.37(0.76-2.47)$ & $1.22(0.66-2.26)$ \\
\hline \multicolumn{7}{|l|}{ Intensity of warfarin use } \\
\hline$\leq 128 \mathrm{DDD} /$ year & 45 & $1.92(1.34-2.74)$ & $1.91(1.34-2.74)$ & $1.31(0.91-1.88)$ & $1.35(0.98-1.86)$ & $1.21(0.89-1.66)$ \\
\hline 128-200 DDD/year & 51 & $1.74(1.24-2.45)$ & $1.77(1.25-2.50)$ & $1.13(0.74-1.74)$ & $0.93(0.57-1.51)$ & $0.94(0.57-1.56)$ \\
\hline$>200$ DDD/year & 25 & $0.73(0.40-1.33)$ & $0.78(0.43-1.43)$ & $0.99(0.58-1.69)$ & $0.97(0.56-1.69)$ & $0.99(0.56-1.77)$ \\
\hline \multicolumn{7}{|c|}{ Warfarin compared to other anticoagulant drugs } \\
\hline Non-warfarin anticoagulant users & 98 & Ref & Ref & Ref & Ref & Ref \\
\hline Warfarin users & 121 & $1.13(0.79-1.61)$ & $1.01(0.71-1.44)$ & $0.93(0.64-1.35)$ & $1.02(0.70-1.48)$ & $0.93(0.65-1.33)$ \\
\hline \multicolumn{7}{|l|}{ Amount of warfarin use } \\
\hline$\leq 200$ DDD & 63 & $1.85(1.25-2.74)$ & $1.63(1.10-2.42)$ & $1.15(0.75-1.77)$ & $1.19(0.78-1.80)$ & $1.06(0.71-1.58)$ \\
\hline 200-667 DDD & 32 & $0.65(0.37-1.14)$ & $0.58(0.33-1.01)$ & $0.68(0.38-1.20)$ & $0.67(0.36-1.25)$ & $0.64(0.34-1.21)$ \\
\hline$>667 \mathrm{DDD}$ & 26 & $0.72(0.39-1.33)$ & $0.67(0.36-1.23)$ & $0.83(0.45-1.55)$ & $1.02(0.56-1.87)$ & $0.87(0.46-1.64)$ \\
\hline \multicolumn{7}{|l|}{ Duration of warfarin use } \\
\hline$\leq 1$ year & 56 & $1.52(1.00-2.31)$ & $1.33(0.88-2.02)$ & $1.00(0.64-1.55)$ & $1.11(0.73-1.68)$ & $0.97(0.65-1.45)$ \\
\hline $2-4$ years & 44 & $0.96(0.61-1.52)$ & $0.85(0.54-1.34)$ & $0.88(0.53-1.46)$ & $0.77(0.44-1.35)$ & $0.78(0.44-1.38)$ \\
\hline 5 or more years & 21 & $0.71(0.35-1.46)$ & $0.69(0.34-1.40)$ & $0.84(0.42-1.67)$ & $1.22(0.64-2.33)$ & $1.02(0.53-2.00)$ \\
\hline \multicolumn{7}{|l|}{ Intensity of warfarin use } \\
\hline$\leq 128 \mathrm{DDD} /$ year & 45 & $1.44(0.93-2.24)$ & $1.25(0.80-1.95)$ & $1.04(0.66-1.64)$ & $1.20(0.78-1.83)$ & $1.02(0.68-1.53)$ \\
\hline 128-200 DDD/year & 51 & $1.31(0.85-2.01)$ & $1.16(0.75-1.78)$ & $0.90(0.54-1.49)$ & $0.82(0.47-1.44)$ & $0.79(0.45-1.40)$ \\
\hline >200 DDD/year & 25 & $0.55(0.29-1.05)$ & $0.51(0.27-0.98)$ & $0.79(0.43-1.44)$ & $0.86(0.46-1.60)$ & $0.83(0.44-1.57)$ \\
\hline
\end{tabular}

Age-adjusted, multivariable-adjusted and lag-time hazard ratios $(95 \% \mathrm{Cl})$ related to all PCa deaths. Statistically significant results are bolded

diagnostic use was not possible. Concordantly, we observed no risk increase for pre-diagnostic warfarin use. Compared to the previous study, we had higher of $\mathrm{PCa}$ deaths and were able to evaluate cumulative amount of post-diagnostic use. We found a risk increase only for some subgroups.

A third study of Park et al. [13] was limited to 247 patients with metastatic $\mathrm{PCa}$ receiving docetaxel chemotherapy, and included only $17 \mathrm{LMWH}$ users and 12 warfarin users. LMWH was associated with an improved survival, whereas warfarin was not. In our larger and more comprehensive study, we found no evidence for improved survival among men using other types of anticoagulants.
In vitro studies have suggested that the coagulation cascade and thrombocytes might be involved in tumor growth and progression, and that anticoagulant drugs could in theory improve prognosis [6-10]. However, in epidemiologic studies, confounding by indication dilutes this potential effect, as advanced cancer is associated with an increased risk of thrombosis and consequently with likelihood of initiating anticoagulant treatment. We controlled for such bias by comparing users of different types of anticoagulants and performing a competing risk analysis. We found no significant risk difference, which does not support the putative beneficial effect of warfarin or any other anticoagulants. 


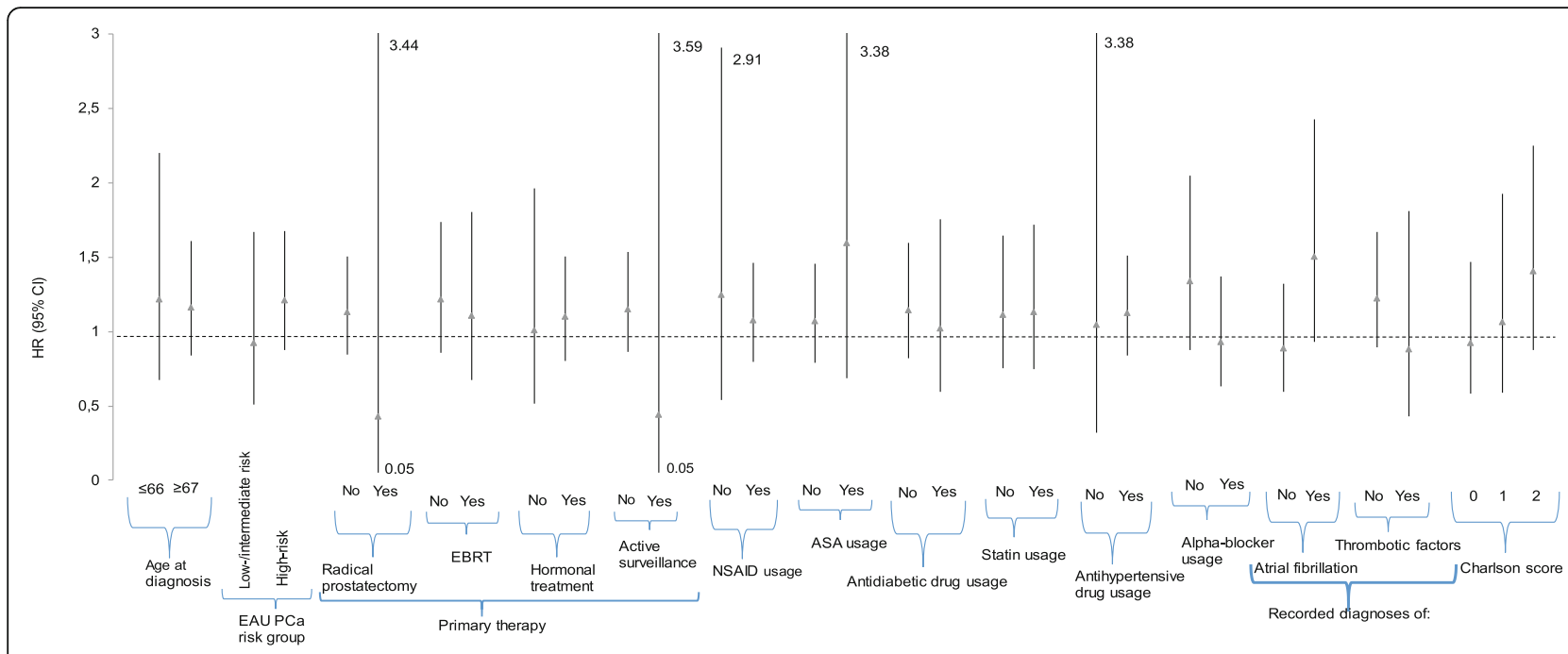

Fig. 1 Pre-diagnostic subgroup analysis of warfarin usage compared to anticoagulant non-users

The main strengths of this study are the large population-based cohort as well as comprehensive and our ability to use detailed, nationally comprehensive register-based information on anticoagulant use unaffected by recall bias. Being able to study large populations through national registries allows us to estimate even relatively uncommonly used drugs such as anticoagulants as cancer risk factor. Our study included substantially more warfarin users than in previous studies. We were able to analyze post-diagnostic use of warfarin more comprehensively than before. We also performed lag-time analyses to estimate the risk associations allowing for latency and removing effects limited to the immediate period following first subscription. Furthermore, we had longer follow-up than in previous studies.
Our study also has some limitations. We were not able to adjust our analysis for smoking, life-style factors or BMI which may be associated with risk of $\mathrm{PCa}$ death [30-33]. Nevertheless, we were able to adjust for Charlson Comorbidity Index and this enabled adjustment for co-existing morbidities. Furthermore, our study was not randomized, and is thus prone to residual confounding.

\section{Conclusion}

In a population-based setting, warfarin or other types of anticoagulants are not associated with improved $\mathrm{PCa}$ prognosis. Conversely, in short-term use risk of $\mathrm{PCa}$ death was increased, which is most likely due to thrombosis caused by an advanced cancer, as the risk increase was not observed in long term.

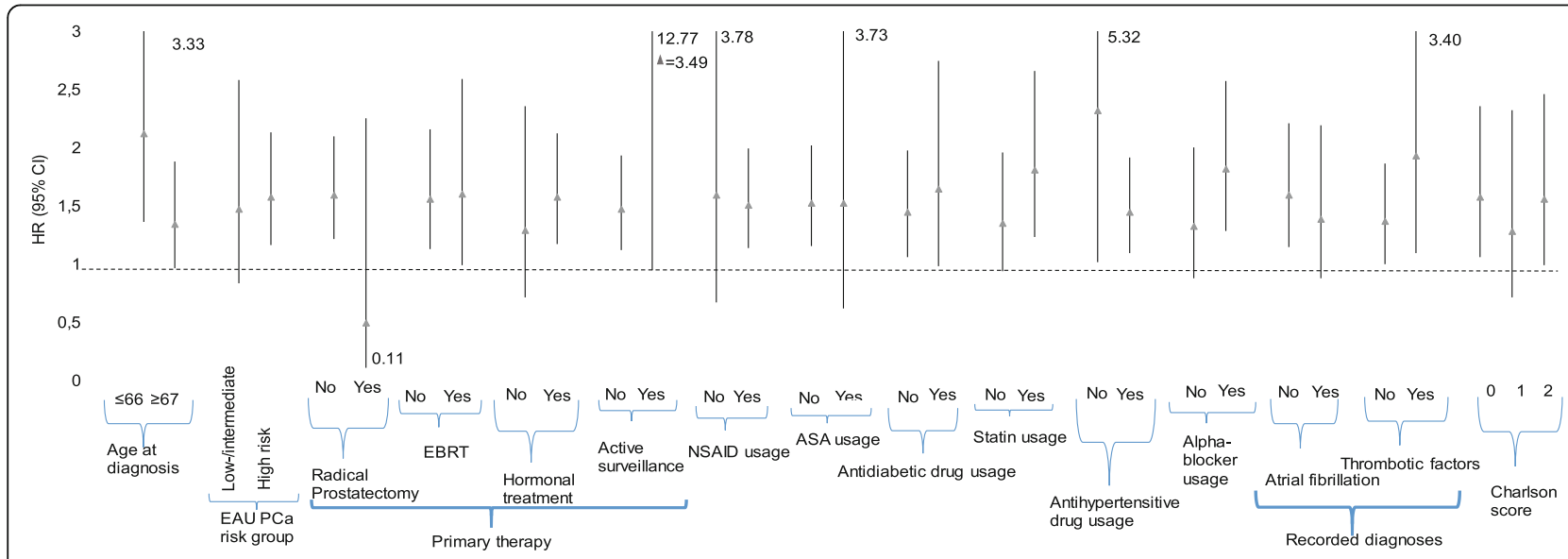

Fig. 2 Post-diagnostic subgroup analysis of warfarin usage compared to anticoagulant non-users 


\section{Additional files}

Additional file 1: Table S1. ATC codes for anticoagulant drugs included in the study. (DOCX $207 \mathrm{~kb}$ )

Additional file 2: Figure S1. Pre-diagnostic subgroup analysis between users of warfarin and other anticoagulant drugs. (DOCX 224 kb)

Additional file 3: Figure S2. Post-diagnostic subgroup analysis between users of warfarin and users of other anticoagulant drugs. (DOCX $224 \mathrm{~kb}$ )

Additional file 4: Table S2. Pre-diagnostic analysis of combined anticoagulant drug usage stratified by number of Defined Daily Doses (DDD), duration and intensity of usage. Age-adjusted and multivariable-adjusted hazard ratios $(95 \% \mathrm{Cl}$ ) related to all PCa deaths. Table S3. Post-diagnostic results for combined anticoagulant usage. Age-adjusted, multivariable-adjusted and lag-time hazard ratios (95\% CI) related to all PCa deaths. (DOCX $405 \mathrm{~kb}$ )

Additional file 5: Figure S3. Subgroup analysis of pre-diagnostic combined anticoagulant usage compared to non-users. (DOCX 224 kb)

Additional file 6: Figure S4. Subgroup analysis of combined anticoagulant usage compared to non-users in post-diagnostic setting. (DOCX $224 \mathrm{~kb})$

\section{Abbreviations}

VTE: Venous thromboembolism; PCa: Prostate cancer; FinRSPC: the Finnish Randomized Study of Screening for Prostate Cancer; EAU: the European Association of Urology; SII: (Finnish) Social Insurance Institution; EBRT: external beam radiation therapy; HR: Hazard ratio; Cl: Confidence interval; DDD: Defined Daily Dose

\section{Acknowledgements}

Not applicable.

\section{Funding}

Supported by non-restricted competitive research funding from the Pirkanmaa Hospital District 150640 to TJ Murtola and non-restricted research grant from Pirkanmaa Cancer Society to PTT Kinnunen. Academy of Finland (grant 132385 and 260 931) and Cancer Organisations of Finland to A Auvinen, Competitive Research funding of Pirkanmaa Hospital District to TLJ Tammela. No Funder organization participated in any part of the study design or collection, analysis or interpretation of data or writing the manuscript.

\section{Availability of data and materials}

Permission to use the entire data has been granted personally for this specific study, thus we are not allowed to make the entire data publicly available without permission. Limited data (i.e variables used for the analyses) can be obtained upon reasonable request.

Each Administrator and Information Commissioner of the used registries processed our request to use the data and granted a personal permission. If desired, similar permissions can be applied from the Finnish Social Insurance Institution (available at: http://www.kela.fi/web/en/research) and the National Institute for Health and Welfare (available at: https://www.thl.fi/en/web/thlfien/statistics/information-for-researchers).

\section{Authors' contributions}

Study Concept: KPTT, MTJ, AA. Study Design: KPTT, MTJ, AA. Data Acquisition: KPTT, MTJ, TK, TK, TTLJ, AA. Quality Control of Data: KPTT, MTJ, TK, TK, TTL, AA. All authors have read and approved the manuscript.

\section{Ethics approval and consent to participate}

The study has been approved by the Ethical Committee of Pirkanmaa Hospital District (Committee's reference number R10167). This study is based on register data collected routinely for other purposes. Thus no informed consent is needed based on international practices.

\section{Consent for publication}

Not applicable.

\section{Competing interests}

We declare the following competing interests:

PTT Kinnunen: none, TJ Murtola: Paid consultant for Astellas and JanssenCilag, lecture fees from Astellas, Janssen-Cilag, Abbvie and MSD, K Talala: none, K Taari: Consulting fee from Abbvie, research funding from Medivation, travel support from Astellas, and Orion, TLJ Tammela: Paid consultant for Astellas, , Orion Pharma and Jansen-Cilag, A Auvinen: lecture fee from MSD, paid consultant for Epid Research Inc.

\section{Publisher's Note}

Springer Nature remains neutral with regard to jurisdictional claims in published maps and institutional affiliations.

\section{Author details}

${ }^{1}$ University of Tampere, Faculty of Medicine and Life Sciences, Tampere, Finland. 'Department of Urology, Tampere University Hospital, Tampere, Finland. ${ }^{3}$ Finnish Cancer Registry, Helsinki, Finland. ${ }^{4}$ Department of Urology, University of Helsinki and Helsinki University Hospital, Helsinki, Finland.

${ }^{5}$ University of Tampere, Faculty of Social Sciences, Tampere, Finland.

Received: 10 April 2017 Accepted: 22 August 2017

Published online: 29 August 2017

References

1. Lyman $\mathrm{GH}$. Venous thromboembolism in the patient with cancer: focus on burden of disease and benefits of thromboprophylaxis. Cancer. 2011 Apr 1;117(7):1334-49.

2. Prandoni $P$, Lensing AW, Prins MR. The natural history of deep-vein thrombosis. Semin Thromb Hemost. 1997;23(2):185-8.

3. Sørensen HT, Mellemkjaer L, Olsen JH, Baron JA. Prognosis of cancers associated with venous thromboembolism. N Engl J Med. 2000 Dec 21;343(25):1846-50

4. Van Hemelrijck M, Adolfsson J, Garmo H, Bill-Axelson A, Bratt O, Ingelsson E, et al. Risk of thromboembolic diseases in men with prostate cancer: results from the population-based PCBaSe Sweden. Lancet Oncol. 2010 May; 11(5):450-8.

5. Chaturvedi S, Sidana S, Elson P, Khorana AA, McCrae KR. Symptomatic and incidental venous thromboembolic disease are both associated with mortality in patients with prostate cancer. PLoS ONE. 2014;9(8):e94048.

6. Maat B. Selective macrophage inhibition abolishes warfarin-induced reduction of metastasis. Br J Cancer. 1980 Feb;41(2):313-6.

7. Brown JM. A study of the mechanism by which anticoagulation with warfarin inhibits blood-borne metastases. Cancer Res. 1973 Jun;33(6):1217-24.

8. Bobek V, Boubelik M, Kovarik J, Taltynov O. Inhibition of adhesion breast cancer cells by anticoagulant drugs and cimetidine. Neoplasma. 2003;50(2):148-51.

9. McCulloch P, George WD. Warfarin inhibition of metastasis: the role of anticoagulation. Br J Surg. 1987 Oct;74(10):879-83.

10. Mousa SA. Anticoagulants in thrombosis and cancer: the missing link. Expert Rev Anticancer Ther 2002 Apr;2(2):227-33.

11. Tagalakis $V$, Tamim $H$, Blostein M, Hanley JA, Kahn SR. Risk of prostate cancer death in long-term users of warfarin: a population-based casecontrol study. Cancer Causes Control. 2013 Jun;24(6):1079-85.

12. O'Rorke MA, Murray LJ, Hughes CM, Cantwell MM, Cardwell CR. The effect of warfarin therapy on breast, colorectal, lung, and prostate cancer survival: a population-based cohort study using the Clinical Practice Research Datalink. Cancer Causes Control. 2015 Mar;26(3):355-66.

13. Park JC, Pratz CF, Tesfaye A, Brodsky RA, Antonarakis ES. The effect of therapeutic anticoagulation on overall survival in men receiving first-line docetaxel chemotherapy for metastatic castration-resistant prostate cancer. Clin Genitourin Cancer. 2015 Feb:13(1):32-8.

14. Ahlbrecht J, Dickmann B, Ay C, Dunkler D, Thaler J, Schmidinger M, et al. Tumor grade is associated with venous thromboembolism in patients with cancer: results from the Vienna Cancer and Thrombosis Study. J Clin Oncol, 2012 Nov 1:30(31):3870-5.

15. Kilpeläinen TP, Tammela TL, Malila N, Hakama M, Santti H, Määttänen L, et al. Prostate cancer mortality in the Finnish randomized screening trial. J Natl Cancer Inst. 2013 May 15;105(10):719-25. 
16. Teppo L, Pukkala E, Lehtonen M. Data quality and quality control of a population-based cancer registry. Experience in Finland. Acta Oncol. 1994;33(4):365-9.

17. EAU risk group stratification, Table 4.2.2. Available at: https://uroweb.org/ wp-content/uploads/EAU-Guidelines-Prostate-Cancer-2016.pdf

18. The Statistical Finland. Available at http://www.stat.fi/index_en.html

19. Martikainen J, Rajaniemi S. Drug reimbursement systems in EU Member States, Iceland and Norway. Helsinki: The Social Insurance Institution, Finland, Social security and health reports. 2002;54 Available at: https://helda.helsinki.fi/handle/10138/13

20. Flahavan EM, Bennett K, Sharp L, Barron TI. A cohort study investigating aspirin use and survival in men with prostate cancer. Ann Oncol. 2014 Jan;25(1):154-9.

21. Holmes S, Griffith EJ, Musto G, Minuk GY. Antihypertensive medications and survival in patients with cancer: a population-based retrospective cohort study. Cancer epidemiol. 2013 Dec;37(6):881-5.

22. Tan P, Wei S, Yang L, Tang Z, Cao D, Liu L, et al. The effect of statins on prostate cancer recurrence and mortality after definitive therapy: a systematic review and meta-analysis. Sci rep. 2016;6:29106.

23. Veitonmäki T, Murtola TJ, Määttänen L, Taari K, Stenman U, Tammela TL, et al. Use of non-steroidal anti-inflammatory drugs and prostate cancer survival in the Finnish prostate cancer screening trial. Prostate. 2015 Sep;75(13):1394-402.

24. Kjellman A, Friis S, Granath F, Gustafsson O, Sorensen HT, Akre O. Treatment with finasteride and prostate cancer survival. Scand J Urol. 2013 Aug:47(4):265-71.

25. Randazzo M, Beatrice J, Huber A, Grobholz R, Manka L, Wyler SF, et al. Influence of metformin use on PSA values, free-to-total PSA, prostate cancer incidence and grade and overall survival in a prospective screening trial (ERSPC Aarau). World J Urol. 2015 Aug;33(8):1189-96.

26. The National Care Register for Health Care maintained by the National Institute for Health and Welfare. Information for reasearchers available at: https:/www.thl.fi/en/web/thlfi-en/statistics/information-for-researchers

27. Charlson ME, Pompei P, Ales KL, MacKenzie CR. A new method of classifying prognostic comorbidity in longitudinal studies: development and validation J Chronic Dis. 1987;40(5):373-83.

28. WHO ATC/DDD index 2016. Available at: http://www.whocc.no/atc_ddd_ index/.

29. Horwitz Rl, Feinstein AR. The problem of "protopathic bias" in case-control studies. Am J Med. 1980;68(2):255-8.

30. Islami F, Moreira DM, Boffetta P, Freedland SJ. A systematic review and meta-analysis of tobacco use and prostate cancer mortality and incidence in prospective cohort studies. Eur Urol. 2014 Dec;66(6):1054-64.

31. Dickerman BA, Markt SC, Koskenvuo M, Pukkala E, Mucci LA, Kaprio J. Alcohol intake, drinking patterns, and prostate cancer risk and mortality: a 30-year prospective cohort study of Finnish twins. Cancer Causes Control. 2016 Sep;27(9):1049-58.

32. Davies NM, Gaunt TR, Lewis SJ, Holly J, Donovan JL, Hamdy FC, et al. The effects of height and BMI on prostate cancer incidence and mortality: a Mendelian randomization study in 20,848 cases and 20,214 controls from the PRACTICAL consortium. Cancer Causes Control. 2015 Nov;26(11):1603-16.

33. Fowke JH, McLerran DF, Gupta PC, He J, Shu X, Ramadas K, et al. Associations of body mass index, smoking, and alcohol consumption with prostate cancer mortality in the Asia Cohort Consortium. Am J Epidemiol. 2015 Sep 1;182(5):381-3.

\section{Submit your next manuscript to BioMed Central and we will help you at every step:}

- We accept pre-submission inquiries

- Our selector tool helps you to find the most relevant journal

- We provide round the clock customer support

- Convenient online submission

- Thorough peer review

- Inclusion in PubMed and all major indexing services

- Maximum visibility for your research

Submit your manuscript at www.biomedcentral.com/submit
Biomed Central 